Aus dem medizinhistorischen Institut der Universität Basel

(Prof. Dr. med. H. Buess)

\title{
Über Spinozas Beitrag zur Leib-Seele-Problematik unter Berücksichtigung der Relation zur modernen Psychosomatik
}

\author{
Von Jürg WunderLI, Zürich
}

Die Barockzeit des 17.Jahrhunderts ist eine Epoche, in welcher auf der ganzen Linie um die gleichen Probleme gerungen wird. Vor allem ist es das Grundproblem des Verhältnisses zwischen Natur und Seele, welches die größten Geister dieser Zeit, Descartes, Leibniz und Spinoza, beschäftigt. Die Mathematik als Ideal höchster, allgemeingültiger rationaler Erkenntnis stand in großer Gunst, und man glaubte, durch übersichtliches Ordnen und Systematisieren an philosophische Probleme die gleiche Beweisführung wie in der Mathematik herantragen zu können. Nicht umsonst hat ja Spinoza sein Hauptwerk, die Ethik, «more geometrico» dargestellt, mit Axiomen, Lehrsätzen, Beweisen, Folge- und Hilfssätzen usw., so daß allein deswegen die Lektüre alles andere als fließend und leicht ist.

Leibniz und Spinoza sind beide auf ihre Art durch die Gedanken ihres Vorgängers René Descartes inspiriert worden. Bekanntlich trennte letzterer in seinem Hauptwerk Principia philosophiae (3) Körper und Seele radikal. Die Innenwelt des Bewußtseins und Denkens (res cogitans) und die äußere, materielle, ausgedehnte Welt (res extensa) sind zwei nicht vergleichbare Wirklichkeiten und Substanzen. Descartes trennte also nicht nur die beiden Aspekte des Seelischen und Körperlichen voneinander, sondern er identifizierte sie mit Substanzen des Seins, führte also einen strengen, nicht nur aspektmäßigen, sondern auch substantiellen Dualismus durch. Das Leibliche stellte sich Descartes als rein mechanistisches Geschehen vor und findet für die Tatsache der Zusammengehörigkeit von Seelischem und Körperlichem im Menschen keine andere Erklärung, als daß hier Gottes Beihilfe angenommen werden müsse. Trotzdem unternimmt er einen schwachen Versuch, die Verknüpfung von Körper und Seele auf einen lokalisierten Sitz im Gehirn zu fixieren. In seinem Traité des passions de l'âme (3) beschreibt er die Zirbeldrüse als Sitz der Seele im Gehirn, 
wobei er sich vorstellt, diese Drüse schwebe oder reite so in der Mitte des Gehirns, daß sie durch die geringste Bewegung der Spiritus, der Lebensgeister, in Bewegung gesetzt werden könne.

Die mittelalterliche Spirituslehre von den - oft spekulativ gedeuteten Vermittlern zwischen Leib und Seele behauptete ohnehin noch ein recht zähes Dasein im Barockzeitalter. Nach MaLPighi (18) sollte die Gehirnrinde diese Spiritus drüsenartig ausscheiden, welch letztere nach Descartes, wie oben erwähnt, das Corpus pineale als Sitz der Seele bewegen. Erst Тномаs Willis vertrat nüchternere Auffassungen, indem er beispielsweise das Großhirn als Organ der willkürlichen Bewegung auffaßte. Die Spiritus animales sind bei Willis nicht mehr "Geister der Lebensseele», sondern ein dynamisches Prinzip, welches in manchem dem damals noch gänzlich unbekannten elektrischen Nervenstrom nahekommt (11).

Der Cartesianismus war ja vor allem den religiösen Ärzten der damaligen Zeit wenig sympathisch (19). Darum kam das Leibnizsche System einer prästabilierten Harmonie zwischen Seele und Leib den Bedürfnissen der Ärzte entgegen. Leibniz wirkte, anders als Spinoza, befruchtend und intensiv auf die Ärzte seiner Zeit (17). Spinoza, als exkommunizierter, gehaßter und verspotteter Jude, in größter Einsamkeit lebend, begann ja erst lange nach seinem Tode auf die Nachwelt zu wirken, insbesondere auf manche geistige Köpfe und Philosophen des letzten Jahrhunderts. Spinoza und Leibniz sind in vielem zu vergleichen: Einerseits besteht eine große Nähe zwischen den beiden, etwa dadurch, daß beide das große Thema des 17. Jahrhunderts, das Verhältnis von Natur und Seele, aufgreifen, anderseits trennte beide doch ein tiefer Graben in der Art und Weise, wie jeder die von ihm konzipierte Einheit von Seele und Natur sieht.

Spinoza wurde am 24. November 1632 in Amsterdam als Sohn von spanischen, nach Portugal vertriebenen und nach Amsterdam emigrierten Juden geboren. Er besuchte die jüdische Schule, studierte Latein, den Talmud und die Bibel in hebräischer Sprache und neben jüdischer Philosophie auch spanische Literatur. Konflikte mit den jüdischen Gelehrten führten 1656 zur Exkommunikation aus der jüdischen Gemeinde, besonders auch, weil er den Synagogenbesuch arg vernachlässigte. Ausgestoßen von den Seinen lebte Spinoza in Einsamkeit, äußerer Abgeschlossenheit und Stille sein einfaches Leben und betrieb neben seinem philosophischen Wirken mit Meisterschaft das Schleifen optischer Gläser. 1662 begann er an seinem Hauptwerk, der Ethik zu arbeiten und feilte bis zu seinem frühen Tod an Tuberkulose, am 21.Februar 1677, daran herum. Noch 1673 hatte 
er eine Berufung nach Heidelberg als Philosophieprofessor abgelehnt, da er trotz der Zusicherung der Lehrfreiheit an letzterer zweifelte.

\section{Spinozas Einsubstanzlehre}

Die Gedanken Spinozas zum Leib-Seele-Problem verdienten es, wieder vermehrt beachtet zu werden. Bevor wir uns aber diesem Thema zuwenden, müssen wir zunächst - als notwendige Voraussetzung - die Einsubstanzlehre streifen. Beides ist in Spinozas Hauptwerk, der Ethik (20), enthalten.

Im Gegensatz zu seinem Vorgänger Descartes betont Spinoza, daß es nur eine Substanz gibt, welche mit Gott gleichzusetzen ist (14. Lehrsatz, Teil I). Diese eine Substanz ist ewig, unendlich, notwendig aus sich selbst existierend und unteilbar (13. Lehrsatz, Teil I). Die göttliche, eine, unteilbare Natur hat unendlich vieles auf unendlich viele Weisen zur Folge (16. Lehrsatz, Teil I). Wichtig ist dabei der Unterschied zwischen der wirkenden Natur (natura naturans) und der gewirkten Natur (natura naturata), der im Scholium zum 29. Lehrsatz des I. Teiles dargestellt wird. Die schaffende natura naturans ist Gott als unendliche, alles hervorbringende Natur, oder anders gesagt, sie stellt die Attribute der einen Substanz dar, welche ewiges und unendliches Wesen ausdrücken. Die geschaffene natura naturata ist demgegenüber der Inbegriff der endlichen Dinge, der endliche Aspekt der einen Substanz, oder, in den Worten Spinozas, sie umfaßt «alle Daseinsformen (Modi) der Attribute Gottes, sofern sie also Dinge betrachtet werden, die in Gott sind und ohne Gott weder sein noch gedacht werden können.» Letztlich und im Eigentlichen sind aber natura naturans und natura naturata identisch, gehören zur einen und einzigen Substanz Gott.

Die unendliche Substanz hat, soweit wir dies wahrnehmen, zwei Attribute: Das Denken (cogitare) und die Ausdehnung (extensio). Spinoza stellt diese, die Gedanken von Descartes weiterführenden Behauptungen, im 1. und 2. Lehrsatz des II.Teiles auf. Gott ist unendliche Ausdehnung, als nicht Körper; denn unter Körper wird gemäß der 1. Begriffsbestimmung des II.Teiles eine Daseinsform (modus) Gottes verstanden, «welche das Wesen Gottes, sofern es als ausgedehntes (räumliches) Ding betrachtet wird, auf gewisse und begrenzte Weise ausdrückt.» Gott ist anderseits auch nicht ein bestimmtes Denken oder ein bestimmtes Bewußtsein (Spinoza setzt Denken und Bewußtsein einander gleich), sondern unendliches Denken. So 
sind die denkende und die ausgedehnte Substanz ein und dieselbe Substanz, bald unter diesem, bald unter jenem Attribut aufgefaßt. Darum ist auch die Daseinsform der Ausdehnung und die Idee dieser Daseinsform ein und dasselbe, nur auf zweierlei Arten ausgedrückt, wie es im Scholium zum 7. Lehrsatz des II.Teiles heißt. Das Identitätsprinzip ist klar aufgestellt.

\section{Seele (Geist) und Körper}

Auf Grund des Bisherigen ist nun das Folgende zu verstehen. Auch die Seele ist kein selbständiges, vom Körper getrenntes Wesen, eine davon verschiedene Substanz, wie es Descartes formuliert hat; Körper und Seele sind nur zwei Aspekte des einen Wesens, welche bald als ausgedehntes, bald als denkendes (mit Bewußtsein ausgestattetes) Ding betrachtet wird. Das Wesen des Menschen kommt aus gewissen Daseinsweisen der Attribute Gottes zustande (Folgesatz zum 10. Lehrsatz des II.Teiles). Der menschliche Geist ist ein Teil des unendlichen Verstandes Gottes. Darauf folgt der berühmte Satz: «Wenn wir also sagen, der menschliche Geist nimmt dieses oder jenes wahr, so sagen wir nichts anderes, als daß Gott - nicht sofern er unendlich ist, sondern sofern er durch die Natur des menschlichen Geistes ausgedrückt wird, oder sofern er das Wesen des menschlichen Geistes ausmacht - diese oder jene Idee hat ...» (Folgesatz zu Lehrsatz 11, II).

Dieser Satz könnte recht ketzerisch und revolutionär anmuten, wird aber bei näherem Zusehen durch die Einschiebung (nicht sofern usw.) erheblich abgeschwächt. Der Körper nun ist Gegenstand der Idee, welche den menschlichen Geist ausmacht (Lehrsatz 13, II). Der Folgesatz zu dem zuletzt erwähnten Lehrsatz ist besonders wichtig, indem hier Spinoza erklärt, daß der Mensch aus Geist und Körper besteht und der menschliche Körper, so wie wir ihn empfinden, existiert. Der menschliche Geist ist mit dem Körper vereinigt, dergestalt, daß die Realität des Körpers zwar betont wird, andererseits der Körper doch nichts anderes ist als der Gegenstand der Idee, des Bewußtseins.

Noch deutlicher drückt sich Spinoza im III. Teil seines Hauptwerkes aus, in welchem im wesentlichen seine bekannte Affektenlehre enthalten ist. Von zentraler Bedeutung ist der zweite Lehrsatz mit dem nachfolgenden Scholium. Danach sind Körper und Geist ein und dasselbe, bald unter dem Attribut des Denkens, bald unter dem Attribut der Ausdehnung gedacht. Strikte wendet sich Spinoza gegen jegliche Wechselwirkung zwischen Geist 
und Körper. Eine solche wäre ja auch undenkbar, wo doch beide lediglich Aspekte der einen Einheit sind. Freilich ist Spinoza selber recht skeptisch, ob er die Menschen einmal dahin bringen werde, vom üblichen Schema der Wechselwirkung abzusehen. Die Menschen sind fest überzeugt, daß sich der Körper auf einen Wink des Geistes bald bewege, bald ruhe, bald viele Handlungen verrichte. Wenn wir aber die Einheit ernst nehmen und folgerichtig jede Wechselwirkung ausschließen, müssen wir auch das Werden von Kunstwerken, Bauten usw. ganz aus dem Körperlichen heraus verstehen, ein Schluß, welcher uns zunächst unsinnig erscheinen mag. Spinoza argumentiert darauf, daß niemand den Körper so genau kenne, daß er alle seine Funktionen erklären könnte. Jene Menschen, welche von Wechselwirkung sprechen, wissen nach seiner Meinung nicht, was sie sagen, hat doch niemand bisher (und dies gilt natürlich bis zum heutigen Tag!) einen genauen Nachweis erbringen können. Spinozas Urteil über solche voreilige Schlüsse ist auch heute noch zu beherzigen, daß man nämlich nichts anderes tut, als mit täuschenden Worten einzugestehen, daß man - ohne darüber in Verwunderung zu geraten - die wahre Ursache jener Tätigkeit nicht weiß.

Die konsequente Aufhebung des Descartesschen Influxus physicus, wie sie schon im 5. und 6. Lehrsatz des II.Teiles eingeleitet wurde, verlangt nach Spinoza von uns, daß wir beispielsweise das, was uns als Sinn verständlich ist und uns im Körperlichen durch den Geist verursacht scheint, körperlich erklären. Dabei müssen wir uns aber bewußt sein, daß man den Begriff «Körper» Spinozas nicht als tote res extensa im Sinne Descartes verstehen darf, sondern als einen beseelten Körper, als modus der einen göttlichen Substanz unter dem Attribut der Ausdehnung, welcher alle Lebensfunktionen - und somit auch die seelischen außer dem Bewußtsein umfaßt. Es ist auch nicht anders als folgerichtig, daß Spinoza vehement Descartes Lehre vom Sitz der Seele abgelehnt hat (im Vorwort zum V.Teil).

Wir wollen kurz zusammenfassen. Geist und Körper sind nur zwei Aspekte desselben Einen. Alle Körper sind geistig und alle Geister sind körperlich. Darum ist es, nebenbei bemerkt, auch grundfalsch, bei Spinoza denselben Mechanismus des Körpers wie bei Descartes anzunehmen, ist der Körper doch immer beseelt. Freilich kann diese Einheit, wie wir später nochmals festhalten werden, nur philosophisch gefaßt werden. Weil der Mensch eines ist, gibt es keine Wechselwirkung zwischen Körper und Seele. Die Einheit besteht; aber die beiden Aspekte sind als solche untrennbar voneinander geschieden. 


\section{Spinoza und das hebräische Menschenverständnis}

Will man Spinoza richtig verstehen, so muß man sich stets vergegenwärtigen, daß er ein Jude war. Nun unterscheidet sich aber gerade das althergebrachte und schon alttestamentlich fixierte hebräische Menschenverständnis ganz erheblich vom griechisch-hellenistischen, wie es noch jahrhundertelang im christlichen Mittelalter weitergewirkt hat. Die griechische Auffassung zeigt eine allgemeine Tendenz zum Dualismus, wie er dann besonders bei Platon zugespitzt wird, etwa wenn Platon vom Leib als Gefängnis der Seele spricht (15). Das Denken der Hebräer ist im allgemeinen monistisch, ganzheitsbezogen und kennt keine scharfe Aufteilung der Funktionen des Menschen (7). Die geistigen Funktionen des Menschen werden nicht von den vitalen körperlichen getrennt. Die alttestamentliche Forschung konnte zeigen, daß das hebräische Wort ,nefeš einfach «Leben »* bedeutet und in der Septuaginta mit «Psyche» falsch übersetzt wurde**.

So ist es nicht erstaunlich, wenn dasselbe, in Richtung Monismus und Einheitsverständnis tendierende Denken bei Spinoza zur fruchtbaren Auseinandersetzung, Antithese und Synthese mit dem extremen Dualismus Descartes führte.

\section{Der psychophysische Parallelismus}

Man hat Spinoza den Vater des psychophysischen Parallelismus genannt und sich dabei hauptsächlich auf den 7. Lehrsatz des II.Teiles gestützt, wo es heißt, daß «die Ordnung und Verknüpfung der Ideen dieselbe ist wie die Ordnung und Verknüpfung der Dinge». Aber man sollte hier etwa vorsichtiger sein und sich vor allem stets vergegenwärtigen, daß es Spinoza ja weniger um ein gleichzeitiges Nebenherlaufen geht, als um den strengen Dualismus - nicht des einen Menschen, nicht von Körper und Seele!-, sondern lediglich der (Forschungs-)Aspekte. Der Gedanke der Einheit ist das Entscheidende, der Dualismus der Aspekte das andere wichtige Moment in Spinozas Denken. Erst G.T.Feghner und später W. Wund, beide im 19. Jahrhundert, haben den eigentlichen psychophysischen Parallelismus begründet. Wundt formuliert diesen in seiner Psychologie in dem Satz, «da $\beta$ alle diejenigen Erfahrungsinhalte, die gleichzeitig der mittelbaren,

* im Sinne des an den Körper gebundenen Lebens.

** Beispielsweise kann der entsprechende Passus im Buch Amos 2, 14-15, übersetzt werden mit: «Sein Leben retten» oder «Sich retten» (13). 
naturwissenschaftlichen und der unmittelbaren, psychologischen Betrachtungsweise angehören, zueinander in Beziehung stehen, indem innerhalb jenes Gebietes jedem elementaren Vorgang auf psychischer Seite ein solcher auf physischer entspricht» (23). G.T.Fechner trug seinen psychophysischen Parallelismus nicht nur mit metaphysischem Schwung vor, sondern er suchte in seiner Psychophysik auch exakt wissenschaftlich die parallelen Beziehungen zwischen Körper und Seele zu ergründen (6).

\section{Zu den Leib-Seele-Theorien der modernen Psychosomatik}

Selbstverständlich ist hier nicht der Ort, um systematisch die Leib-SeeleTheorien der unzähligen Richtungen moderner Psychosomatik zu erörtern. Es geht nur darum, Gedankengänge zu finden, die in etwa mit denjenigen Spinozas korrespondieren können. Wir fragen vor allem nach psychosomatischen Forschern, welche sich gegen das übliche Schema der psychophysischen Wechselwirkung wenden; denn es ist ja tatsächlich eine bei näherem Zusehen unhaltbare, weil klischeehafte Vorstellung, daß beides, ein Leib und eine Seele, existieren und beide nun aufeinander wechselseitig einwirken - ohne daß man vom Wesen dieser «Einwirkung» auch nur die Spur einer Ahnung hat. Besonders der Daseinsanalytiker M.Boss (2) lehnt in seiner Einführung in die psychosomatische Medizin vehement die übliche Kausalkette des technischen Weltbildes (Psyche - Hirnstamm - vegetative Dystonie - anatomische Strukturläsion im Organ - organische Krankheit) ab. Freilich ist seine Ausdrucksweise, welche sich an HeIDEgGers Existentialphilosophie anlehnt, sonderbar und oft schwer verständlich, und man kann sich fragen, ob das ganze nichts anderes als eine bloße Theorie ist; «denn $\mathrm{da} \beta$ es der wesensmäßige Lebensauftrag des Menschen sei, seine Existenz auszutragen, kann entweder geglaubt werden oder nicht» (12).

Wohl der erste, welcher das Verhältnis von Leib und Seele mit einem ähnlichen Problem der modernen Physik verglichen hat, ist G. R. Heyer (9).

In einem Vortrag im September 1927 über «Das Quantenpostulat und die neuere Entwicklung der Atomistik» führte BoHr den Begriff der Komplementarität ein und verstand darunter zwei Bilder, die sich gegenseitig ausschließen, aber doch notwendig ergänzen (21). Das Licht kann sowohl als eine Korpuskularstrahlung als auch als eine Wellenbewegung aufgefaßt werden. In gleicher Weise imponieren die Elektronen je nach Forschungsmethode bald als Materialwellen, bald als kleinste Materiepartikelchen (8). 
Der Dualismus der beiden Beschreibungen ist unvereinbar; jeder Aspekt schließt den anderen aus. Andererseits ist eine vollständige Beschreibung der Einheit «Elektron» oder «Licht» erst möglich, wenn beide Forschungsaspekte berücksichtigt werden. Diese Komplementarität übertrug Heyer auf das Leib-Seele-Problem, und v. Eickstedt (5) führte diesen Gedanken weiter, indem er sich bemühte, aufzuzeigen, daß für das Atomare und das Psychische ähnliche Gesetzmäßigkeiten gelten - demnach eine neuartige Form von Psychophysik.

Von besonderer Bedeutung ist aber die sogenannte Äquivalenztheorie von F.Alverdes (1). Psychisches und Physisches sind demnach zwei aequivalente Ausdrucksweisen einer dahinterstehenden Einheit. Die wirkliche Beschaffenheit der Einheit kann nicht erkannt werden. Ein menschlicher Lebensvorgang erscheint uns, wenn er mit chemisch-physikalischer Forschungsmethodik untersucht wird, als somatisch, mit psychologischer Methodik aber als rein psychisch. Dieser Dualismus ist jedoch nur scheinbar und beruht lediglich auf der Begrenztheit unserer Forschungsmethoden und menschlichen Erkenntnismöglichkeiten. Es fehlt uns gewissermaßen der archimedische Punkt, um das Ganze überblicken zu können. Daher ist auch die Wechselbeziehung zwischen Soma und Psyche ein bloßer Scheinverhalt; denn zwei Erscheinungsformen eines an sich einheitlichen Lebensvorganges können nicht aufeinander wirken.

Wir sehen, daß wir mit solchen modernen Gedanken in größte Nähe zu den Auffassungen Spinozas gelangen, der auch in anderer Hinsicht als Vorläufer der modernen Psychosomatik bezeichnet werden kann (14).

\section{Kritische Beleuchtung von Spinozas Gedanken}

Erstens. Vom philosophischen Standpunkt aus ist es richtig, wenn wir Leib und Seele als Einheit sehen. Spinoza wehrt sich dagegen, daß der Mensch als Geistwesen der Natur gegenübergestellt wird. Der Mensch ist, wenn man es so ausdrücken darf, zugleich ein Stück Natur - wo Natur wiederum weit mehr bedeutet als lediglich den Inbegriff des naturwissenschaftlich Erfaßbaren -, und zwar sowohl der gezeugten natura naturata wie der selbst hervorbringenden natura naturans. Wir müssen jedoch betonen, daß solche Ganzheitsauffassung nur rein philosophisch sein kann, da wir mit den empirischen Methoden niemals die Ganzheit als solche, sondern lediglich gewisse Aspekte erfassen können. 
Zweitens. Diese Erkenntnis, daß wir nie das Ganze empirisch erfassen können, sondern nur die einzelnen Aspekte, oder wie Spinoza sagen würde, bald unter dem Attribut des Denkens, bald unter dem Attribut der Ausdehnung, muß auch in Spinoza aufgeleuchtet haben. Gerade darum wendet er sich gegen einen lokalisierten Sitz im Gehirn. Gerade darum hält er bei aller letzlichen Identität am Dualismus der Attribute - oder, wie wir sagen würden, der Aspekte und Methoden - fest. Seine Gedanken entsprechen hierin in erstaunlicher Weise der modernen Áuffassung von der Komplementarität.

Drittens. Aus seinem strengen Dualismus der Attribute und aus der Lehre von der letztlichen, philosophischen Einheit heraus hat Spinoza jegliche Wechselbeziehung geleugnet. Daran ist heute noch richtig, daß unser Reden von der Wechselwirkung stets irgendwie in der Luft hängt, weil wir sie nie direkt nachweisen können. Wir stellen ja bestenfalls lediglich die Gleichzeitigkeit von physischen und psychischen Phänomenen fest. Auch die Entdeckung etwa der Bedeutung des limbischen Systems für die affektive Steuerung hat wohl unser Bild von der somatischen Seite erweitert, aber keinen Übergang vom Soma zur Psyche gezeigt. Freilich wäre, wie JASPERS (10) schreibt, zu fragen, ob Spinoza nicht von der modernen Psychologie und Psychosomatik aus zu erweitern wäre, indem die praktische Forschung nicht nur die strenge Trennung der beiden Aspekte berücksichtigen muß, sondern beispielsweise «Greifbarkeiten ins Auge faßt, die in der Erscheinung zugleich leiblich und geistig sind (Sprache, Ausdruck)».

Viertens. Spinozas System hat - wie jedes noch so geniale System - seine Lücken und Unklarheiten. Beispielsweise wird der Leib beseelt gedacht, andererseits aber doch der Seele oder dem Geist mit dem Bewußtsein - als Attribut des Denkens - gegenübergestellt. Oder: Spinoza betont die Realität des Körpers, betrachtet aber den letzteren als nichts anderes als den Gegenstand der Idee. So können wir fragen, wie sich denn der Zweifel beseitigen lasse, ob es nicht doch außerhalb des Bewußtseins keine Existenz des Körpers gebe, denselben Zweifel, welchen Descartes in seinem System nur mühsam und unter Zuhilfenahme eines Deus ex machina aus der Welt schaffen konnte.

Über solche Lücken und Unklarheiten muß man hinwegsehen können. Es geht darum, daß wir die Hauptlinien in Spinozas Denken richtig erkennen, wird letzteres doch nur dann für uns fruchtbar, wenn wir sein System nicht noch systematischer machen und verabsolutieren wollen. Nach diesen Hauptlinien besteht eine letztliche und eigentliche Identität - gibt es doch 
nur einen Gott, nur eine Substanz - die, um es nochmals zu betonen, mit einer Substanz im materiellen Sinne nichts zu tun hat, sondern einem unbeschreibbaren Ganzen und Einen entspricht, aus welchem alles entstammt und in welches alles einmündet, nur den einen Menschen als leibseelische Einheit. Aller Dualismus ist nicht eigentlich, nicht letztlich, sondern beruht einzig auf der begrenzten menschlichen Anschauung, welche nur Aspekte erkennen kann. Diese Erkenntnis ist entscheidend und auch in der Gegenwart nicht weniger gültig als zu Spinozas Zeiten.

\section{Zusammenfassung}

Spinozas philosophisches System kann in mancher Beziehung als fruchtbarer Vorläufer der modernen Psychologie betrachtet werden. Besonders aufschlußreich - aber heute vielleicht etwas zu wenig beachtet - sind seine Auffassungen zur Leib-Seele-Problematik.

Spinoza faßt Ausdehnung und Denken als Attribute der einen «Natura» auf, die man aber sehr zu Unrecht mit einer materiellen Substanz gleichsetzt, indem man Spinoza zu den materialistischen Monisten zählt. Es geht ihm darum, Leib und Seele als zwei grundverschiedene Aspekte der einen Einheit darzustellen. Diese Lehre wird im folgenden mit einigen modernen psychosomatischen Theorien verglichen.

\section{Literaturverzeichnis}

1. Alverdes F., Die Theorie der psycho-physischen Äquivalenz (Das Leib-Seele-Problem) Studium Generale, 1954, S. $21 \mathrm{ff}$.

2. Boss M., Einführung in die psychosomatische Medizin, Huber, Bern 1954.

3. Descartes R., Euvres et lettres, textes présentés par André Bridoux, Bibl. de la Pléiade, Paris 1949.

4. Diepgen P., Geschichte der Medizin in drei Bänden, de Gruyter, Berlin 1949-1955.

5. v. Eickstedt E., Atom und Psyche, Enke, Stuttgart 1954.

6. Fechner G.T., In Sachen Psychophysik, von Breitkopf, Leipzig 1877.

7. Fiorenza Francis P. und Metz Johann Baptist, Der Mensch als Einheit von Leib und Seele, in Mysterium salutis, Band 2, Benziger, Einsiedeln 1967.

8. Heisenberg W., Physik und Philosophie, Ullstein-Taschenbücher, 1959.

9. Heyer G.R., Vom Kraftfeld der Seele. Zwei Abhandlungen zur Tiefenpsychologie, Klett, Stuttgart 1949.

10. Jaspers K., Aus dem Ursprung denkende Metaphysiker, Kap. Spinoza, S. $144 \mathrm{ff}$,, Piper, München 1954. 
11. IsLer H., Thomas Willis, ein Wegbereiter der modernen Medizin, Wissenschaftliche Verlagsgesellschaft, Stuttgart 1965.

12. Lutz St., Zur Psychopathologie von 6 an Ulcus duodeni resp. ventriculi leidenden Kindern und Jugendlichen. Diss. Zürich 1967.

13. MaAg Viktor, Text, Wortschatz und Begriffswelt des Buches Amos, Brill, Leiden 1951.

14. Meerloo J.A.M., Spinoza: Coup d'œil sur les conceptions psychologiques. Médecine et Hygiène 23 (1965) 255-256.

15. Platon, Phaidon 62 b, in Hauptwerke, herausgegeben von W.Nestle, Kröner, Stuttgart 1950.

16. Regensburg J., Über die Abhängigkeit der Seelenlehre Spinozas von seiner Körperlehre. Diss. Riga 1900.

17. Rотнsснuн K.E., Die Beziehungen zwischen Leibniz und der Medizin seiner Zeit, Hippokrates 21 (1966) 864/870.

18. Rотнsснuн K.E., Geschichte der Physiologie, Springer, Berlin 1953.

19. Rотнsснин K. E., Descartes und die Theorie der Lebenserscheinungen, Sudhoffs Arch. 50 (1966) 25.

20. Spinoza, Die Ethik (und andere Schriften), herausgegeben von F. BüLow, Kröner, Stuttgart 1966.

21. Weizsäcker C.F. von, Zum Weltbild der Physik, 10. Auflage, S. Hirzel, Stuttgart 1961, S. $281 \mathrm{ff}$.

22. WolfF H., Spinozas Ethik, eine kritische Einführung, Francke, Bern, 1958.

23. Wundt W., Grundriß der Psychologie, 12.Auflage, Kröner, Leipzig 1914. 